\title{
Topological $\mathrm{BCL}^{+}-$algebras
}

\section{Yonghong Liu}

School of Automation, Wuhan University of Technology, Wuhan, China

\section{Email address: \\ hylinin@163.com}

\section{To cite this article:}

Yonghong Liu. Topological BCL ${ }^{+}$- Algebras. Pure and Applied Mathematics Journal. Vol. 3, No. 1, 2014, pp. 11-13. doi: $10.11648 /$ j.pamj.20140301.13

\begin{abstract}
BCL} / \mathrm{BCL}^{+}$-algebras $[1,2]$ are more extensive class than $\mathrm{BCK} / \mathrm{BCI} / \mathrm{BCH}$-algebras [5-8], introduced by Yonghong Liu. In this paper, we aim to embed topological structure in $\mathrm{BCL}^{+}$-algebras, thus we should be introduced to the notion of "good taste", which is both topological space and metric space. Thereupon, some fundamental properties of topological $\mathrm{BCL}^{+}$-algebras are obtained.
\end{abstract}

Keywords: $\mathrm{BCL}-A \operatorname{lgebra}, \mathrm{BCL}^{+}$-Algebra, Topological $\mathrm{BCL}^{+}$-Algebra

\section{Introduction}

In [1], Yonghong Liu introduced a new class of abstract algebra: BCL-algebra. Recently, Yonghong Liu introduced $a$ wide class of abstract algebras: $\mathrm{BCL}^{+}$-algebra (see [2, 4]). In [3], Deena Al-Kadi and Rodyna Hosny introduced the deformation of BCL-algebra. They continue to investigate the relation between BCL-algebra and $\mathrm{d} / \mathrm{BCH} / \mathrm{BCI}$ and BCK-algebra.

In this paper we are going to introduce topological spaces for the $\mathrm{BCL}^{+}$-algebras, which is derived the fundamental properties, which we will need the following definitions and theorems:

\section{Definition 1.1 [2]}

An algebra $(X ; *, 1)$ is called a $\mathrm{BCL}^{+}$-algebra if it satisfies the following laws hold: for any $x, y, z \in X$,

$\left.\mathrm{BCL}^{+}-1\right) \quad x * x=1$.

$\left.\mathrm{BCL}^{+}-2\right) \quad x * y=1$ and $y * x=1$ imply $x=y$.

$\left.\mathrm{BCL}^{+}-3\right)((x * y) * z) *((x * z) * y)=((z * y) * x)$.

\section{Theorem 1.1 [2]}

Assume that $(X ; *, 1)$ is any a $\mathrm{BCL}^{+}$-algebra. Then the following hold: for any $x, y, z \in X$

i) $(x *(x * y)) * y=1$.

ii) $x * 1=x$ imply $x=1$.

iii) $((x * y) *(x * z)) *(z * y)=1$.

iv) $\left.\mathrm{BCL}^{+}-2\right) x * y=1$ and $y * x=1$ imply $x=y$.

\section{Theorem 1.2 [2]}

An algebra $(X ; *, 1)$ is a $\mathrm{BCL}^{+}$-algebra if and only if it satisfies the following conditions: for all $x, y, z \in X$
i) $\left.\mathrm{BCL}^{+}-1\right) \quad x * x=1$.
ii) $\left.\mathrm{BCL}^{+}-2\right) \quad x * y=1$ and $y * x=1$ imply $x=y$.
iii) $(((x * y) * z) *((x * z) * y)) *((z * y) * x)=1$.
iv) $x *(1 * y)=x$.

\section{Definition 1.2 [2]}

Suppose that $(X ; *, 1)$ is a $\mathrm{BCL}^{+}$-algebra, the ordered relation if $x \leq y$ if and only if $x * y=1$, for all $x, y \in X$, then $(X ; \leq)$ is partially ordered set and $(X ; *, 1)$ is an algebra of partially ordered relation.

\section{Corollary 1.1 [2]}

Let every $x \in X$. Then 1 (one) is maximal element in a $\mathrm{BCL}^{+}$-Algebra $(X ; *, 1)$ such that integral $1 \leq x$ imply $x=1$.

\section{Main Results}

For a $g(x, y, z)=(x * y) * z$-algebra, the topological space is just a basic concept in nature. We have the following definitions.

\section{Definition 2.1}

Let $(X ; \circ, *, 1)$ be a $\mathrm{BCL}_{D}^{+}$-algebra with two binary 
operations $\circ$ and $*$ that satisfies the following properties: for any $x, y, z \in X$.

$\left.\mathrm{BCL}_{D}^{+}-1\right)$ An algebra $D(X)=(X ; \circ)$ is a distributive algebra.

$\left.\mathrm{BCL}_{D}^{+}-2\right) \quad$ An algebra $P(X)=(X ; *, 1)$ is a

$g(x, y, z)=(x * y) * z$-algebra.

$\left.\mathrm{BCL}_{D}^{+}-3\right)$ (right weakly distribution)

$x *(y \circ z)=(x * y) \circ(x * z)$.

$\left.\mathrm{BCL}_{D}^{+}-4\right)$ (left weakly distribution)

$(y \circ z) * x=(y * x) \circ(z * x)$.

\section{Definition 2.2}

Let $(X ; *, 1)$ be a $\mathrm{BCL}^{+}$-algebra and let $(X, \mathfrak{I})$ be a topological space. If

$$
\circ, *: X \times X \rightarrow X \text { and }(x, y) \mapsto x * y
$$

be a continuous mapping from the product space $(X \times X, \mathfrak{R})$ to the topological space $(X, \mathfrak{I})$, where $\mathfrak{R}$ is product topology of $X$, then we say that $(X ; \mathfrak{I}, *, 1)$ is a topological $\mathrm{BCL}^{+}$-algebra.

\section{Theorem 2.1}

The product of any tow topological $\mathrm{BCL}^{+}$-algebra is again a topological $\mathrm{BCL}^{+}$-algebra.

Proof: Let $\left(X_{1} ; \mathfrak{I}, *_{1}, 1_{1}\right)$ and $\left(X_{2} ; \mathfrak{I}, *_{2}, 1_{2}\right)$ be topological $\mathrm{BCL}^{+}$-algebra, let $\left(x_{1}, y_{1}\right)$ and $\left(x_{2}, y_{2}\right)$ is any tow element in $X$, and defines:

$$
\left\{\begin{array}{l}
X=X_{1} \times X_{2} \\
\circ:\left(x_{1}, y_{1}\right) \circ\left(x_{2}, y_{2}\right)=\left(x_{1} *_{1} x_{2}, y_{1} *_{2} y_{2}\right) \\
1=\left(1_{1}, 1_{2}\right)
\end{array}\right.
$$

Now suppose that $U$ is a open neighborhood of $\mathfrak{R}$. By Definition 2.2, $\Re$ be product topology, so we conclude that $B_{1}$ is an open neighborhood of $x_{1} *_{1} x_{2}$, and $B_{2}$ is an open neighborhood of $y_{1} *_{2} y_{2}$ in $X$, we have $V_{1} \times V_{2} \subseteq U$. Since $B_{1}$ is an open neighborhood of $x_{1}$, and $B_{2}$ is an open neighborhood of $x_{2}$. Also, $B_{3}$ is a open neighborhood of $y_{1}$, and $B_{4}$ is a open neighborhood of $y_{2}$, we have

$$
B_{1} * B_{2} \subseteq V_{1} \text { and } B_{3} * B_{4} \subseteq V_{2} .
$$

Then

$$
\left(B_{1} * B_{2}\right) \circ\left(B_{3} * B_{4}\right) \subseteq V_{1} \times V_{2} \subseteq U,
$$

and we claim that in fact

$$
\left(B_{1} * B_{2}\right) \times\left(B_{3} * B_{4}\right)=\left(B_{1} \times B_{3}\right) \circ\left(B_{2} \times B_{4}\right) \subseteq U,
$$

where $B_{1} \times B_{3}$ is a open neighborhood of $\left(x_{1}, y_{1}\right)$ in $X$, and $B_{2} \times B_{4}$ is a open neighborhood of $\left(x_{2}, y_{2}\right)$ in $X$. Thus $\circ$ is a continuous and $(X ; \mathfrak{I}, \circ, 1)$ is a topological $\mathrm{BCL}^{+}$-algebra.

\section{Corollary 2.1}

The product of any finite topological $\mathrm{BCL}^{+}$-algebra is again a topological $\mathrm{BCL}^{+}$-algebra.

\section{Corollary 2.2}

Any finite product of a topological $\mathrm{BCL}^{+}$-algebra is again a topological $\mathrm{BCL}^{+}$-algebra.

\section{Example 2.1}

Let $X$ be a topological $\mathrm{BCL}^{+}$-algebra and let $f(x, y, z)$ and $g(x, y, z)$ be ternary polynomial. Assume that for all $x, y, z \in X$. Suppose the following conditions hold:
i) $f(x, y, z)=((x * y) * z) *((x * z) * y)$, and
ii) $g(x, y, z)=(z * y) * x$.
If $(f(x, y, z), g(x, y, z))=1$, then we say that $f(x, y, z)$ and $g(x, y, z)$ are coprime topological $\mathrm{BCL}^{+}$-algebras.

\section{Example 2.2}

Let $X$ be a topological $\mathrm{BCL}^{+}$-algebra and let $f(x, y, z)$, $g(x, y, z)$ and $h(x, y, z)$ be ternary polynomial. Assume that for all $x, y, z \in X$. Suppose the following conditions hold:

i) $f(x, y, z)=(z * y) * x$.

ii) $g(x, y, z)=(x * y) * z$.

iii) $h(x, y, z)=(x * z) * y$.

If $(f(x, y, z), g(x, y, z))=1$ and $(f(x, y, z), h(x, y, z))=1$.

Then

$$
(f(x, y, z), g(x, y, z) * h(x, y, z))=1 .
$$

\section{Definition 2.3}

If $\mathrm{BCL}_{D}^{+}$-algebra $(X ; \circ, *, 1)$ has the property of distribution, let $\mathfrak{I}$ be topology of $X$ and let $(X ; \mathfrak{I}, \circ, *, 1)$ be a topological $\mathrm{BCL}_{D}^{+}$-algebra. Then topological $\mathrm{BCL}_{D}^{+}$-algebra $(X ; \mathfrak{I}, \circ, *, 1)$ has also the property of distribution.

\section{Theorem 2.2}

Let $(X ; \circ, *, 1)$ be a $\mathrm{BCL}_{D}^{+}$-algebra and let $\mathfrak{I}$ be topology of $X$, then $(X ; \mathfrak{I}, \circ, *, 1)$ be a topological $\mathrm{BCL}_{D}^{+}$ - algebra. 
Proof: Let the operations o

$$
\circ:\left(x_{1}, y_{1}\right) \circ\left(x_{2}, y_{2}\right)=\left(x_{1} * x_{2}, y_{1} * y_{2}\right) \text {. }
$$

identically, element $1=(1,1)$. By Definition 2.1 and Definition 1.3, we have continuous mapping

$$
\circ, *:(X \times X, \Re) \rightarrow(X, \mathfrak{I}) \text {. }
$$

where $\Re$ is product topology of $X$. We deduce that $(X ; \mathfrak{I}, \circ, *, 1)$ be a topological $\mathrm{BCL}_{D}^{+}$-algebra.

\section{Definition 2.4}

Let $(X ; \mathfrak{I}, \circ, *, 1)$ be a topological $\mathrm{BCL}_{D}^{+}$-algebra. Suppose the following conditions hold: for any $x, y, z \in X$.

i) $\mathfrak{I}(x, y) \geq 1$ and $\mathfrak{I}(x, y)=1$ if and only if $x=y$.

ii) $\mathfrak{I}(x, y)=\mathfrak{I}(y, x)$.

iii) $\mathfrak{I}(x, z) \leq \mathfrak{I}(x, y)+\mathfrak{I}(y, z)$.

Then $(X, \mathfrak{I})$ be a metric space of identically element 1 .

\section{Example 2.3}

Let $X$ be a set and let $\mathfrak{I}: X \times X \rightarrow \mathbb{R}, x, y \in X$, by Definition 2.3, we now have

$$
\mathfrak{I}(x, y)= \begin{cases}1 & \text { if } x=y \\ 0 & \text { if } x \neq y\end{cases}
$$

Then $\mathfrak{I}$ be a discrete metric and $(X, \mathfrak{I})$ be a discrete metric space.

\section{Theorem 2.3}

Let $(X ; \circ, *, 1)$ be a $\mathrm{BCL}_{D}^{+}$-algebra and let $\mathfrak{I}$ a discrete topology of $X$, then $(X, \mathfrak{I})$ be a topological space.

Proof: By Theorem 2.1, $(X, \mathfrak{I})$ be a discrete metric space by Example 2.1, where discrete metric of $X$ is induced by $\mathfrak{I}$. Thus $(X, \mathfrak{I})$ be a topological space.

\section{References}

[1] Y. H. Liu, "A New Branch of the Pure Algebra: BCL-Algebras", Advances in Pure Mathematics, vol. 1, no. 5, pp. 297-299, 2011.

[2] Y. H. Liu, "On BCL ${ }^{+}$-Algebras", Advances in Pure Mathematics, vol. 2, no. 1, pp. 59-61, 2012.

[3] A. K. Deena and H. Rodyna, "On BCL-Algebra”, Journal of Advances in Mathematics, vol. 3, no. 2, pp. 184-190, 2013.

[4] Y. H. Liu, "Partial Orders in $\mathrm{BCL}^{+}$-Algebra", Journal of Advances in Mathematics, vol. 5, no. 1, pp. 630-634, 2013.

[5] Y. Imai and K. Iséki, "On Axiom System of Propositional Cal-culi XIV," Proceedings of the Japan Academy, vol. 42, no. 1, pp. 19-22, 1966.

[6] K. Iséki, "An Algebra Related with a Propositional Calculus," Proceedings of the Japan Academy, vol. 42, no. 1, pp. 26-29, 1966.

[7] K. Iseki, "On BCI-Algebras," Mathematics Seminar Notes (Kobe University), vol. 8, no. 1, pp. 125-130, 1980.

[8] Q. P. Hu and X. Li, "On BCH-Algebras," Mathematics Seminar Notes (Kobe University), vol. 11, no. 2, pp. 313-320, 1983. 